\title{
La religion laïque de George Santayana comme un mode de vie
}

George Santayana laizistische Religion als Lebenspraxis

George Santayana's lay religion as a way of life

\section{Nóra Horváth}

Traducteur : Eva Rouault

\section{(2) OpenEdition}

Journals

Édition électronique

URL : http://journals.openedition.org/ceg/3103

DOI : $10.4000 /$ ceg. 3103

ISSN : 2605-8359

Éditeur

Presses Universitaires de Provence

Édition imprimée

Date de publication : 26 avril 2018

Pagination : 135-150

ISBN : 979-10-320-0160-8

ISSN : 0751-4239

Référence électronique

Nóra Horváth, "La religion laïque de George Santayana comme un mode de vie », Cahiers d'Études Germaniques [En ligne], 74 | 2018, mis en ligne le 26 octobre 2019, consulté le 26 novembre 2020.

URL : http://journals.openedition.org/ceg/3103; DOI : https://doi.org/10.4000/ceg.3103 


\title{
La religion laïque de George Santayana comme un mode de vie
}

\author{
Nóra Horváth \\ Université Széchenyi István Faculté Apáczai, Győr, Hongrie
}

$\mathrm{Au}$ milieu du XX $\mathrm{X}^{\mathrm{e}}$ siècle, George Santayana a reconsidéré sa philosophie comme une philosophie laïque (lay religion) dont les origines remontent à l'Antiquité : " ma philosophie est, comme celles des Anciens, une discipline de l'esprit et du cœur, une religion laïque ${ }^{1}$ ". Les croyants ont du mal à accepter l'attitude constamment critique de Santayana vis-à-vis des religions traditionnelles, néanmoins, d'un point de vue philosophique, l'une des problématiques les plus intéressantes que soulève son œuvre, ainsi que sa conduite de vie, concerne l'équivalence substantielle de la poésie et de la religion. C'est avant tout ce rapprochement de la poésie et de la religion qui me semble contenir un horizon d'actualité pour la religion. C'est avec l'objectif de mettre au jour les dimensions de sa réflexion sur la religion qui conservent un intérêt pour le monde contemporain que je souhaite présenter, dans mon étude, les sentiments personnels qu'exprime Santayana à l'égard de la religion, et je veux examiner ses écrits sur la religion afin d'illustrer que sa pensée sur la religion et sur les enjeux religieux reste éminemment moderne.

Si nous voulons avoir une image complète de sa relation à la religion, il semble essentiel d'examiner non seulement ses écrits mais aussi toute sa vie dans la mesure où, pour Santayana, la philosophie est un mode de vie et non pas seulement une discipline académique ou théorie scientifique. Or, ce positionnement de la philosophie débouche par quasi-nécessité sur des interrogations qui entrent dans le champ du religieux et - comme nous allons le voir - de la poésie.

Je pense que la meilleure façon d'examiner l'œuvre de Santayana est de le faire dans le cadre de l'esthétique de l'existence ou des systèmes notionnels de sa philosophie de l'art de vivre ${ }^{2}$. Outre sa philosophie individualiste, qui a pour but l'harmonie et la célébration des sentiments, Santayana s'est sérieusement préoccupé de l'envahissement intolérant de toute sorte de pouvoirs. Santayana a accordé une attention particulière aux questions du fanatisme, et j'estime

1. "My philosophy is like that of the ancients a discipline of the mind and heart, a lay religion ", in George Santayana, The Realm of Spirit, New York, Scribner, 1940, p. 272.

2. Cf. Nóra Horváth, "Lovers of Beauty" - The CEuvres of George Santayana, Fred Holland Day and Edward Perry Warren as Exemplification of Aesthetics of Existence Influenced by the Platonic Eros ", Brno Studies in English, 41/2, 2015, p. 5-21. 
d'ailleurs que ses vues sur le fanatisme religieux pourraient alimenter les débats philosophiques d'aujourd'hui. Dans mon analyse, j'utilise les écrits autobiographiques de Santayana et je présente son ouvrage Interpretations of Poetry and Religion, car il est la source de sa théorie de la poésie comme religion. Pour défınir la notion de l'imagination, je fais référence à The Sense of Beauty, et parmi les courts essais je mentionne aussi Natural and Ultimate Religion. Mais le propos de mon étude se concentre plus particulièrement sur l'ouvrage de Santayana qui s'intitule Reason in Religion: il est son interprétation la plus importante du rôle de la religion dans la société et me semble pouvoir être une contribution de valeur pour le débat au XXI ${ }^{\mathrm{e}}$ siècle.

À la question de savoir qui, selon lui, deviendrait le philosophe le plus lu parmi ses contemporains à l'avenir, Alfred North Whitehead avait répondu: George Santayana ${ }^{3}$. Même si aujourd'hui nombreux sont ceux qui s'étonnent de cette déclaration, dans la première partie du $\mathrm{XX}^{\mathrm{e}}$ siècle, la carrière d'écrivain de Santayana était à son apogée. Son autobiographie, Persons And Places (1944), et son unique roman, The Last Puritan (1936) étaient devenus des best-sellers, ce dernier étant même nommé pour le prix Pulitzer, et son autobiographie complète en trois volumes était comparée aux ouvrages littéraires les plus marquants de l'époque. Son œuvre est extrêmement variée, révélatrice de centres d'intérêt très diversifiés. Selon Newton P. Stallknecht, les théories développées par Santayana dans sa réflexion philosophique ne sont pas caractérisées par une originalité radicale. Dans l'histoire académique de la philosophie américaine, il reçoit moins d'attention et occupe une place plus modeste que ses contemporains, William James, C. S. Pierce ou John Dewey. Cependant, si nous considérons l'œuvre intégrale de Santayana, sa grande ouverture d'esprit et la multitude des genres littéraires qu'il a empruntés, alors nous nous retrouvons face à une richesse qui n'existe chez aucun autre penseur de notre siècle. Stallknecht considère Santayana comme " le plus grand humaniste " de son époque ${ }^{4}$, dont l'œuvre est caractérisée - malgré son naturalisme contradictoire - par le refus et la critique de la civilisation occidentale, et par la recherche de la synthèse entre l'éthique grecque et chrétienne.

Il défend l'idée de la réalisation de soi et soutient le talent de l'individu. "En réalité, dès qu'il touche quelque chose, il le transforme en philosophie ${ }^{5}$ ", a écrit de lui Stallknecht. On ne peut pas décrire la réflexion philosophique de Santayana à travers les catégories habituelles de la philosophie. Il n'appartenait à aucune école philosophique. Il a refusé l'étiquette de pragmatiste, et en réalité, on ne peut évidemment pas le considérer comme pragmatiste mais, grâce à son ontologie esthétique, il peut néanmoins être considéré comme précurseur de l'esthétique pragmatiste. Dans la majorité de ses œuvres, nous pouvons

3. Irving Singer, George Santayana Literary Philosopher, New Haven / London, Yale University Press, 2000, p. 7.

4. Newton P. Stallknecht, George Santayana, Minneapolis, University of Minnesota Press, 1971, p. 6.

5. Ibid., p. 13. 
constater qu'il était naturaliste, et pourtant c'est la philosophie de Platon qui a le plus influencé ses thèses sur la beauté, l'amour et l'essence. Il était catholique espagnol, mais la plupart du temps il se considérait comme aesthetic Catholic et Catholic atheist. Il vivait parmi les habitants puritains de Boston et, dans ce milieu protestant, s'est senti étranger et exclu.

\section{La tentative d'un mode de vie esthétique}

La philosophie de Santayana a servi sa propre libération spirituelle (spiritual liberation). Après avoir quitté sa chaire à l'Université en 1912, il a, selon ses propres dires, privilégié la vie spirituelle plutôt que la carrière et, renonçant au “système”, s'est créé un monde pour lui-même. On ne peut pas le considérer comme philosophe argumentatif. Son style est un style littéraire personnel, mais il pratique la philosophie au sens noble du terme, par amour de la sagesse. Dans l'histoire de la philosophie américaine il est le plus engagé pour clamer l'importance de la "spiritual life ". La Spiritual Life est une des notions les plus contestées dans la philosophie de Santayana, sujette à des explications contradictoires, qui donnent des points de vue souvent complètement opposés sur sa spiritualité. Daniel Moreno, un chercheur espagnol, a désigné la vie spirituelle comme élément essentiel de la philosophie de Santayana dans Santayana The Philosopher - Philosophy As a Form of Life, ouvrage paru au début des années $2000^{6}$. Cependant, dans cette vie-là, il ne s'agit pas de la spiritualité au sens religieux, mais davantage de la célébration des intuitions qui sont toujours intimement liées à la notion d'essence (essence) dans la philosophie de Santayana. Ses propos laissent supposer qu'intuitivement, l'individu peut sortir des difficultés du monde ordinaire et peut s'adonner à un état contemplatif qui laisse paraître la vérité derrière les apparences. À mon avis, c'est à travers son analyse de l'œuvre de Marcel Proust que la théorie de Santayana sur la notion d'essence est le plus aisément compréhensible. Son court essai, intitulé Proust on Essences révèle le mieux l'importance de l'émergence instantanée des essences ${ }^{7}$. L'apparition soudaine d'une essence est toujours un moment rare et précieux, complètement indépendant de la volonté de l'individu. Ces instants qui dénoncent quelque chose ou expriment une cohérence plus profonde, ne reviennent jamais. Dans son essai, Santayana cite de nombreux extraits du Temps retrouvé; ces citations sont plus parlantes que toute autre explication. Santayana évoque le narrateur du roman de Proust au moment où il prend conscience du sens de sa vie et de sa mission: "Aussi cette contemplation de 1 'essence des choses, j'étais maintenant décidé à m'attacher à elle, à la fixer ${ }^{8}$. " La concentration instantanée du passé et du présent, de manière indépendante $d u$

6. Daniel Moreno, Santayana The Philosopher - Philosophy As a Form of Life, Lewisburg, Bucknell University Press, 2015, p. 145.

7. George Santayana, " Proust on Essences ", in Essays in Literary Criticism, éd. par Irving Singer, New York, Charles Scribner's Sons, 1956, p. 245.

8. Ibid., p. 243. 
temps, provoque un sentiment de réjouissance. Le narrateur du roman de Proust décide à cet instant précis de consacrer toute sa vie à coucher ses contemplations sur le papier - bien évidemment, ce n'est pas un hasard si Santayana a placé précisément ces passages au cœur de ses réflexions. Écrire, créer une œuvre, ont aussi conduit Santayana, en tant qu'auteur, à repenser et revivre sa vie. Santayana a passé la deuxième partie de sa vie à Rome, cloîtré dans un hospice, en se consacrant à l'écriture mais sans pratiquer sa religion. C'est par l'écriture qu'il a créé son identité spirituelle.

Dans ses confessions intitulées The Idler and His Works, Santayana a réinterrogé de manière approfondie l'intégralité de son œuvre. Il a eu le sentiment que son parcours peut être examiné de deux points de vue: d'une part poétique, d'autre part théorique. Il reconnaît pouvoir trouver quelquefois des " touches poétiques et des envols irresponsables ${ }^{9}$ " dans ses travaux scientifiques, de la même façon que des problématiques sérieuses apparaissent dans sa poésie. " Mes vers et mes réflexions intimes m'appartenaient, m'exprimaient et ne s'adressaient essentiellement à personne d'autre. Les questions académiques m'ont été suggérées et imposées par les circonstances et j’apparais ici dans le costume et sous le masque d'un caractère assumé ${ }^{10}$ ", a-t-il écrit. Irving Singer - un des plus grands spécialistes de l'œuvre de Santayana - l'a décrit comme un philosophe littéraire dans son ouvrage paru en 2000. Selon Singer, Santayana a réussi à fusionner l'écriture littéraire et philosophique ${ }^{11}$. La célèbre assertion selon laquelle Santayana était un " poète - philosophe " et non pas un " philosophe - poète " provient de Richard Butler ${ }^{12}$. Santayana a considéré que la réflexion philosophique est fondamentalement une activité littéraire ${ }^{13}$. Il ne parvenait jamais à distinguer fermement au fond de lui l'activité de poète de celle de philosophe. Il écrit ainsi dans la préface d'un de ses recueils de poèmes:

For as to the subject of these poems, it is simply my philosophy in the making. [...] I see no reason why a philosopher should be puzzled. What he sees he sees; of the rest he is ignorant; and his sense of this vast ignorance (which is his natural and inevitable condition) is a chief part of his knowledge and of his emotion. Philosophy is not an optional theme that may occupy him on occasion. It is his only possible life ${ }^{14}$.

9. "Poetic touches and irresponsible flights ", in George Santayana, Daniel Cory, The Idler and His Works, New York, G. Braziller, 1957, p. 5.

10. "My verses and my private philosophizing belonged to me, expressed me, and were addressed essentially to nobody else; the academic subjects were suggested or imposed by circumstances, and I appear there in the costume and under the mask of an assumed character ", in George Santayana, Daniel Cory, The Idler and His Works, New York, G. Braziller, 1957, p. 4.

11. Irving Singer, George Santayana Literary Philosopher, p. 2.

12. Richard Butler, The Life and World of George Santayana, Chicago, A Gateway Edition, Henry Regnery Company, 1960, p. 98.

13. Irving Singer, George Santayana Literary Philosopher, p. 2.

14. "Concernant le sujet de ces poèmes, c'est tout simplement ma philosophie en devenir. [...] Je ne vois pas pourquoi un philosophe devrait être embarrassé. Il voit ce qu'il voit, il ignore le reste ; et le sens de sa profonde ignorance (qui est sa condition naturelle et inévitable), est la partie majeure de sa connaissance et de son émotion. La philosophie n'est pas un sujet optionnel qui puisse l'occuper de temps en temps. C'est sa seule vie possible ", in George Santayana, Poems, Preface, London/Bombay/Sydney, Constable and Company Ltd., 1922, p. xii-xiii. 
Santayana n'était pas croyant. Comme il l'a confié dans son autobiographie: " Je n'ai jamais pratiqué ma religion ou cru pouvoir grâce à elle gagner le paradis ou éviter l'enfer [...] Tout ce qu'il s'est passé, c'est que je me suis habitué à une Weltanschauung différente, à une autre sorte de système qui a la même fonction rationnelle que la religion: me garder attentif aux leçons de la vie ${ }^{15}$." Cette autre sorte de Weltanschauung était la " vital philosophy " de Santayana, une approche critique, " modest Epicureanism " ou une sorte de stoïcisme. Il revendique son éclectisme comme condition de son ouverture d'esprit. Il conclut dans General Confession: " on ne peut faire confiance à aucun système ${ }^{16}[. .$.$] ".$ Ce point de vue explique son refus total de la violence des systèmes religieux mais aussi le rejet de la notion de valeurs absolues. Selon Santayana il n’y a ni valeur, ni idéal absolu, ni image de Dieu ou même une vérité absolue, car il considère que les nouvelles expériences que l'humanité peut vivre sont capables de transcender les idéaux précédents. Pour lui, une seule chose est absolue: la créativité de l'imagination.

Pour Santayana, le passé a toujours été très important et il a vénéré les bâtiments, les lieux, qui incarnaient les valeurs du passé. Même si dans sa jeunesse il n'avait pas encore une vision globale de l'histoire de l'architecture, il était attiré par les formes originales, qu'il prenait plaisir à admirer. Les églises et les rues d'Avila ont déterminé toute sa vie, et ses méditations sur les événements du passé ont rempli sa vie à tel point qu'il ne s'est jamais senti seul, ou qu'il n'a pas vécu sa solitude de façon négative. Sa passion à entrer dans les églises dans sa jeunesse revêt un aspect esthétique. Il a écouté la messe en langue étrangère, éprouvé du plaisir à la musique et au chant des hommes, bien qu'il n'y prît aucune part. Il est resté spectateur de l'extérieur. Mais il a toujours ressenti le besoin de l'atmosphère des églises, de la lumière colorée des vitraux, de la majesté des bâtiments: " J'ai respiré plus librement dans l'atmosphère de la religion que dans celle du monde des affaires car la religion comme la poésie est plus idéale, et libère davantage l'imagination ${ }^{17}$ " a-t-il affırmé. Une des grandes passions de la jeunesse de Santayana était son admiration absolue pour les églises gothiques. Il était fasciné par l'ancienne tapisserie murale de l'église d'Avila, une œuvre monumentale de couleur rouge, dans laquelle étaient accumulés des souvenirs séculaires. Puis, la tapisserie fut acquise par un millionnaire américain contre vingt mille dollars, une offre que

15. "I had never practised my religion, or thought of it as a means of getting to heaven or avoiding hell [...] All that happened was that I became accustomed to a different Weltanschauung, to another system having the same rational function as religion: that of keeping me attentive to the lessons of life ", in George Santayana, My Host the World, New York, Charles Scribner's and Sons, 1953, p. 4.

16. " No system is to be trusted [...]; but all systems may be used and, up to a certain point, trusted as symbols ", George Santayana, General Confessions, p. 9 in Paul Arthur Schilpp (dir.), The Philosophy of George Santayana, Chicago/Evanston, Northwestern University, 1940, p. 495-606.

17. "I breathed more easily in the atmosphere of religion than in that of business, precisely because religion, like poetry, was more ideal, more freely imaginary ", in George Santayana, Persons and Places, The Background of My Life, New York, Charles Scribner's Sons, p. 174-175. 
les élus d'Avila ne pouvaient pas refuser. L'explication donnée: " elle était déjà très abîmée " était complètement inacceptable pour Santayana, qui se rappelait cette magnifique église comme la dernière " où il a ressenti la plénitude de l'âme en écoutant la messe ${ }^{18}$ ". Il a aimé la quiétude fraîche des églises et le chatoiement de leur vitraux ${ }^{19}$. Déjà jeune garçon, il était attiré par l'architecture, surtout après la lecture de l'ouvrage de Ruskin : Les Pierres de Venise. Quand il a quitté les États-Unis pour l'Allemagne, il s'est arrêté en chemin pour visiter des cathédrales françaises et espagnoles. Il a écrit alors: "Il me paraissait que l'art devait être plus réel que la nature, qu'autrement il perdait sa raison d'être ${ }^{20}$. Par "plus réel", j'entends plus primitif, plus simple et plus clair... ${ }^{21}$ ".

Il a visité presque chaque jour les musées où il s'est promené parmi les statues de marbre grecques, mais aussi les peintures allemandes et italiennes ${ }^{22}$. Il était avide de sentiments, non de connaissances. Il était empli de bonheur quand dans une église ou en écoutant la musique, il parvenait à des associations d'idées qu'il n'aurait pas connues autrement.

C'est seulement dans son œuvre tardive, dans My Opinions où Santayana a résumé ses principales pensées, qu'il a exprimé clairement le but de sa philosophie: être la célébration des sentiments qui envahissent l'homme dans les moments contemplatifs.

The arts may die of triviality, as they were born of enthusiasm. On the other hand, there will always be beauty, or a transport akin to the sense of beauty, in any high contemplative moment. And it is only in contemplative moments that life is truly vital, when routine gives place to intuition, and experience is synthesised and brought before the spirit in its sweep and truth. The intention of my philosophy has certainly been to attain, if possible, such wide intuitions, and to celebrate the emotions with which they fill the mind. If this object be aesthetic and merely poetical, well and good: but it is a poetry or aestheticism which shines by disillusion and is simply intent on the unvarnished truth ${ }^{23}$.

Santayana a contesté l'autonomie de l'esthétique (comme science). Toute tentative d'isoler une théorie esthétique de l'ensemble de son œuvre rendrait

18. Ibid., p.112-113.

19. Ibid., p.114-115.

20. En français dans le texte original.

21. "Art, it seems to me, must be more real than nature, or it loses its raison d'être. By more real, I mean more primitive, simple and clear ». Lettre de G.S. à H. W. Abbot du 12 décembre 1886, Berlin, in John McCormick, George Santayana a biography, New York, Alfred A. Knopf, 1987, p. 59.

22. Ibid., p. 60.

23. « La trivialité peut tuer les arts, car ceux-ci sont nés de l'enthousiasme. D’autre part, il y aura toujours de la beauté, ou un transport comparable au sens de la beauté, dans chaque moment hautement contemplatif. Et c'est seulement dans ces moments contemplatifs que la vie est primordiale, quand la routine laisse la place à l'intuition, et quand l'expérience est synthétisée et amenée avant l'esprit dans sa totale vérité. Le but de ma philosophie est d'atteindre, si possible, des intuitions aussi profondes, et de célébrer les émotions avec lesquelles elles remplissent l'esprit. Si cet objectif est esthétique et purement poétique, soit! Mais dans ce cas, c'est une poésie ou un esthétisme illuminé par la désillusion et qui se porte uniquement sur la vérité sans fard ", in George Santayana, A Brief History of My Opinions, in Irwin Edman, The Philosophy of Santayana, New York, Random House, The Modern Library Edition, 1942, p. 21. 
incompréhensible son mode de vie esthétique. Le sens de la beauté et la sensibilité esthétique ont une importance capitale pour lui car il pense qu'ils déterminent nos jugements et influencent notre réflexion. À mon avis, pour comprendre et apprécier l'œuvre de Santayana, l'approche philosophique de son art de vivre serait le plus efficace et le rapprocherait aussi de la philosophie contemporaine. En ce qui concerne ses pensées sur l'auto-érudition et l'auto-création ${ }^{24}$, nous pouvons les analyser non seulement en référence aux œuvres philosophiques anciennes mais aussi sous l'angle des théories actuelles de Richard Rorty ou Richard Shusterman ${ }^{25}$.

\section{La religion comme poésie, la poésie comme philosophie}

En nous basant sur les premières œuvres de Santayana, nous pouvons constater qu'au sujet de la religion il utilise deux points de vue: d'une part, il glorifie le caractère poétique de la religion, d'autre part il essaie d'expliquer, de la façon la plus naturelle, les motivations de la pensée religieuse. Néanmoins, sa conclusion est toujours la même: il suggère que la religion est la plus grande aide pour les hommes parce qu'elle peut donner de l'espoir en toutes circonstances. En même temps, il donne du fil à retordre à ses spécialistes en affirmant que la religion et la poésie ont la même racine et qu'elles sont deux expressions de la célébration de la vie: «Religion et poésie sont identiques en substance, et diffèrent seulement dans la façon dont elles sont rattachées aux affaires pratiques. La poésie s'appelle religion quand elle intervient dans la vie, et la religion quand elle s'ajoute simplement à la vie, apparaît comme n'étant rien d'autre que poésie ${ }^{26}$. "

Dans Interpretations of Poetry and Religion, Santayana a fait paraître des écrits qui étaient déjà publiés dans les colonnes de différentes revues. Santayana désigne la réflexion contemplative comme l'inspiration de la poésie et de la religion, dont le principal vecteur est l'imagination. C'est uniquement l'imagination qui fait naître des idées larges et des harmonies profondes écrit-il, et il considère, que "l'imagination doit fournir à la religion et à la métaphysique des idées larges teintées de passion, des formes suprasensibles, enveloppées dans l'admiration ${ }^{27}$ ".

Nous pouvons affirmer que l'intuition et l'imagination sont les deux notions les plus déterminantes de sa philosophie première, qu'il met plus particulièrement

24. Self-cultivation and self-creation.

25. Cf. Richard Rorty, Contingency, Irony and Solidarity, Cambridge University Press, 1989. Richard Shusterman, Practicing Philosophy: Pragmatism and the Philosophical Life, New York, Routledge, 1997.

26. "Religion and poetry are identical in essence, and differ merely in the way in which they are attached to practical affairs. Poetry is called religion when it intervenes in life, and religion, when it merely supervenes upon life, is seen to be nothing but poetry ", in George Santayana, Interpretations of Poetry and Religion, New York, Charles Scribner's Sons, 1927, p. V.

27. "Imagination must furnish to religion and to metaphysics those large ideas tinctured with passion, those supersensible forms shrouded in awe, in which alone a mind of great sweep and vitality can find its congenial objects " (ibid., p. 6). 
en exergue dans The Sense of Beauty en 1896. Pour Santayana, l'imagination est la création spontanée de la raison qui peut être plus vivante que la réalité. Selon la conception de Santayana, à l'aide de notre imagination et de nos associations, nous pouvons nous créer un niveau intellectuel plus élevé. Chez Santayana, l'activité créative de l'imagination génère les plus grandes valeurs esthétiques. À un âge avancé, Santayana avait l'habitude de dire que la religion est le jeu de l'imagination et citait les mots d'Agustin Santayana qui lui a toujours répété que la religion est comme les poèmes. Lucrèce, Dante et son père ont aussi influencé le développement de son esprit ${ }^{28}$. Il se souvient ainsi dans sa confession intitulée A Brief History of My Opinions:

I have never had any unquestioning faith in any dogma, and have never been what is called a practising Catholic. Indeed, it would hardly have been possible. My mother, like her father before her, was a Deist: she was sure there was a God, for who else could have made the world? But God was too great to take special thought for man: sacrifices, prayers, churches, and tales of immortality were invented by rascally priests in order to dominate the foolish. My father, except for the Deism, was emphatically of the same opinion. Thus, although I learned my prayers and catechism by rote, as was then inevitable in Spain, I knew that my parents regarded all religion as a work of human imagination: and I agreed, and still agree, with them there. But this carried an implication in their minds against which every instinct in me rebelled, namely that the works of human imagination are bad. No, said I to myself even as a boy: they are good ${ }^{29}$.

Les pensées de Santayana sont reflétées par les vers de son disciple, le célèbre poète Wallace Stevens qui a recherché " la littérature suprême " dans le monde qui transcende la religion. "Quand l'homme ne croit plus en Dieu, alors la littérature doit s'y substituer comme rédempteur de la vie. [...] j’ai un tel sentiment concernant le monde, que rien ne peut le satisfaire, à part la poésie. ${ }^{30}$ "Santayana, tout comme Stevens étaient influencés par la thèse la plus importante du point de vue de l'histoire culturelle, la théorie de Matthew Arnold, selon laquelle la poésie prendra la place de la religion. George Howgate affirme dans son ouvrage consacré à Santayana que nous pouvons déjà trouver chez Matthew Arnold une formulation quasi identique à celle de Santayana dans

28. McCormick, George Santayana a biography, p. 45.

29. “ Je n'ai jamais cru de manière absolue à un dogme, et je n'ai jamais été ce qu'on appelle un catholique pratiquant. Cela aurait été difficilement possible. Ma mère comme son père avant elle, était déiste: elle était sûre de l'existence de Dieu, car qui d'autre aurait pu créer le monde? Mais Dieu était trop parfait pour avoir une pensée particulière pour les hommes: les sacrifices, les prières, les églises et les histoires sur l'immortalité étaient inventées par les méchants prêtres pour dominer les imbéciles. Mon père, - excepté le déisme - avait catégoriquement la même opinion. Ainsi, même si j'ai appris les prières et le catéchisme par cœur, comme c'était inévitable à l'époque en Espagne, je savais que mes parents regardaient toute religion comme le résultat de l'imagination humaine; et j'étais d'accord et suis toujours d'accord avec eux là-dessus. Mais cela a eu des conséquences dans leur esprit contre lesquelles je me suis rebellé, notamment que les œuvres de l'imagination humaine sont mauvaises. Non ! - me suis-je dit alors que j'étais encore un jeune garçon, elles sont bonnes ", Santayana, A Brief History of My Opinions, in Edman, The Philosophy of Santayana, p. 5.

30. Richard Ruland, Malcolm Bradbury, Az amerikai irodalom története, Budapest, Corvina, 1997, p. 272. 
son rapport au luxe, à l'industrie, à l'athlétisme, et en général à la vie ${ }^{31}$. Selon Howgate, Santayana est proche d'Arnold, d'un point de vue théorique, parce que celui-ci l'a beaucoup influencé dans sa jeunesse, mais il est vrai aussi que dans ses œuvres tardives il observe l'héritage d'Arnold avec un certain recul critique. L'enthousiasme d'Arnold pour la proclamation de la poésie comme prophétie, son mépris à l'égard du philistinisme, sa profonde estime pour la Grèce antique, mais aussi l'utilisation de l'ironie et le lyrisme de son raisonnement renvoient à l'œuvre de Santayana ${ }^{32}$.

Dans son essai comparant le sentiment religieux païen et chrétien, Matthew Arnold a parlé de la raison imaginative comme d'une nouvelle forme contemporaine de la religion, qui, dans sa période la plus aboutie, est apparue dans la poésie, comme par exemple dans les œuvres de Sophocle, Pindare et Eschyle. Il a pensé que la poésie de son époque devrait aussi suivre ces grands prédécesseurs $^{33}$, car seules les grandes œuvres littéraires sont capables de pénétrer l'homme et d'organiser ses pensées, ses sentiments dans un ensemble harmonieux. Il a caractérisé la transformation réalisable par l'éducation comme une évolution noble, dont le moyen principal peut être la littérature, qui prend petit à petit la succession de la religion et de la philosophie. Une des thèses les plus importantes de la théorie d'Arnold, selon laquelle la poésie prendra la place de la religion, a connu au début du $\mathrm{XX}^{\mathrm{e}}$ siècle une résurgence chez Santayana, lequel, dans ses premières œuvres esthétiques, a aussi régulièrement évoqué le triptyque poésie - religion - philosophie ${ }^{34}$. Dans la deuxième moitié $\mathrm{du} \mathrm{XX}^{\mathrm{e}}$ siècle, cette même pensée revient clairement dans la philosophie de Richard Rorty, en tant que théorie de la culture littéraire comme substitution à la religion et à la philosophie. C'est dans son étude Philosophy as a Transitional Genre qu'il a présenté sa théorie selon laquelle les intellectuels occidentaux ont traversé trois stades depuis la Renaissance: premièrement c'est de Dieu qu'ils ont attendu la rédemption, puis de la philosophie, et maintenant, de la littérature ${ }^{35}$. Tandis qu'aux yeux de Rorty la faute de la philosophie est de s'être éloignée des gens, qui pour cette raison se sont tournés vers les œuvres littéraires plus accessibles, c'est la poésie philosophique que Santayana, dans Three Philosophical Poets, a placée au sommet de la poésie. Donc, leurs motivations et leurs opinions divergent. Les critiques de Santayana n'ont pas toujours apprécié son engagement pour la littérature, et la place d'honneur de " philosophe littéraire " qu'il aurait méritée dans l'histoire de la philosophie ne lui a pas été accordée. Les débats philosophiques contemporains ont créé le contexte

31. George W. Howgate, George Santayana, Philadelphia, University of Pennsylvania Press, 1938, p. 52.

32. Ibid.

33. Matthew Arnold, Pagan and Medieval Religious Sentiment, p. 221, in Arnold, Essays in Criticism, London, Macmillan and Co., St. Martin's Street, 1911, p. 194-223.

34. John Henry Raleigh, Matthew Arnold and American Culture, Berkeley Los Angeles, University of California Press, 1961, p. 155.

35. Richard Rorty, Philosophy as a Transitional Genre, p. 1-28. Cf. [https://mitpress.mit.edu/sites/ default/files/titles/content/9780262524278_sch_0001.pdf]. 
qui présente d'un nouveau point de vue la relation étroite entre la poésie et la philosophie dans une œuvre. Richard Shusterman, par exemple, dans son livre paru en 1997 Practicing Philosophy: Pragmatism and the Philosophical Life ${ }^{36}$, consacre un sous-chapitre à la thématique "philosophical living as aesthetic ". Il y présente le rôle privilégié de la poésie dans la tradition philosophique, selon laquelle la vie devrait être façonnée comme une œuvre d'art. Selon Shusterman, à côté de la voix prophétique de Friedrich Nietzsche et de Charles Baudelaire, nous pouvons entendre celle de Ludwig Wittgenstein, au XX $\mathrm{XX}^{\mathrm{e}}$ siècle, dont la vision esthétique sur la vie et sur la philosophie est aussi associée à la poésie. Même si Shusterman ne le mentionne pas, il serait fascinant de comparer les pensées de Santayana et de Wittgenstein sur la composition poétique. Dans Culture and Value, Wittgenstein affirme: "Je pense avoir résumé mon rapport à la philosophie quand j'ai dit: la philosophie devrait être écrite seulement comme une composition poétique ${ }^{37}$. " Le questionnement de Santayana semble rejoindre cette réflexion dans Three Philosophical Poets : " Est-ce que les poètes, au fond de leur cœur, sont à la recherche de la philosophie? Ou la philosophie n'est-elle rien d'autre, finalement, que poésie ${ }^{38}$ ? " Plus tard, en résumant l'essence de la contemplation imaginative, il constate qu'elle n'est accessible qu'à ceux qui élargissent leur horizon et brident l'élan de leur cœur. C'est ainsi qu'un philosophe peut devenir poète ou un poète potentiellement philosophe, mais uniquement celui qui est capable de faire preuve d'une imagination passionnée dans une sorte de système global élargi ${ }^{39}$.

Aux yeux de Santayana, la fonction noble de la poésie est de saisir la véracité des sentiments cachés sous la surface des idéaux ordinaires. Selon lui, le poète reconstruit ses images dans le but d'en créer l'harmonie. Pour lui, le poète dont l'imagination n'est pas en complète harmonie ni ses expériences composées dans la même symphonie, n'est pas parfait. La conscience de l'étendue de son monde caractérise le poète unique, inégalable. S'il est vraiment sans pareil, sa vision - comme celle de Dante - veut toujours "atteindre les étoiles " ". Santayana considère que dans l'esprit d'Homère et de Dante, les expériences fragmentaires forment un ensemble, une image parfaite, car leur univers est total. Homère vit dans le système de la religion grecque, tandis que Dante vit dans celui de la religion catholique. Il considère au contraire que le monde de Shakespeare n'est pas religieux car il n'est pas construit selon un cadre défini et inaliénable. Selon Santayana, l'attention de Shakespeare était attirée par le monde ordinaire, le monde de la passion et de la beauté. La valeur de son art

36. Richard Shusterman, Practicing Philosophy: Pragmatism and the Philosophical Life, New York, Routledge, 1997.

37. " I think I summed up my attitude to philosophy when I said: philosophy ought really to be written only as a poetic composition ", Ludwig Wittgenstein, Culture and Value, Oxford, Blackwell, 1980, p. 4-5.

38. " Are poets, at heart, in search of a philosophy? Or is philosophy, in the end, nothing but poetry? "Santayana, Three Philosophical Poets, p. 8.

39. Santayana, Three Philosophical Poets, p. 11.

40. George Santayana, The Elements and Functions of Poetry, in Santayana, Interpretations, p. 275. 
réside dans l'imagination triomphante. Le mérite de Shakespeare est de créer un système unique par son pouvoir imaginatif qui n'est ni chrétien, ni païen, mais majestueux grâce à son unité conceptuelle, ce qui constitue un mérite esthétique ${ }^{41}$.

Je considère que le sens esthétique, l'émerveillement au monde et la création d'un univers propre ont plus de valeur pour Santayana que l'appartenance à toute religion et que l'expérience d'une croyance incontestable. Sa foi dans les valeurs supérieures s'exprime dans la quête de la perfection et de la connaissance, tel le désir contenu chez Socrate. Mais Santayana ne croit pas en l'existence des idées de façon autonome. De plus, son approche naturaliste concernant son sens de la beauté ne permet pas de le considérer comme idéaliste.

\section{The Life of Reason et l'espoir à surpasser le chaos}

Dans une de ses œuvres les plus importantes, The Life of Reason, parue en cinq tomes en 1905-1906, Santayana, observe la raison humaine (human reason) dans une approche naturaliste. Il a affirmé " qu'on appelle "life of reason" chaque pensée pratique et action justifiées par leurs propres résultats dans la conscience $^{42} "$. La vie de la raison (the life of reason) est un processus actif, une forme de vie, un moyen pour l'harmonie. Santayana s'efforce de soutenir dans cette œuvre que l'homme est capable de sélectionner les idéaux qu'il va suivre et les impulsions auxquelles il va obéir. "Life of Reason " est la conception d'une vie harmonieuse qui aide à parfaire la connaissance de soi et le savoir. Cette théorie ne refuse pas les passions mais préconise la retenue.

Dans plusieurs de ses œuvres, Santayana a affırmé ne pas détenir de recette qui permettrait d'atteindre une vie heureuse et parfaite, car les hommes et les circonstances de leurs vies sont très différents. Santayana insiste sur le fait que, en dirigeant la raison, tout le monde est capable de choisir ses buts et ses idéaux. Nous pouvons lire dans The Sense of Beauty qu' " aucun athéisme n'est aussi terrible que l'absence d'un idéal ultime ${ }^{43}$ ". Par conséquent, même l'athéisme ne peut être pire que la situation où l'individu n'est pas capable de définir par lui-même l'ordre de valeur moral qui pourrait être son idéal ultime.

Le troisième volume de The Life of Reason est Reason in Religion. Dans cette partie Santayana affirme que dans la vie rationnelle, la religion apparaît aussi comme un facteur déterminant et que la "Life of reason " est un idéal en soi auquel il faudrait tout subordonner dans le monde. Mais l'essence de la religion n'est pas la même que celle de la raison. Dans The Reason in Religion, Santayana remarque que tandis que la religion est constituée d'espoir

41. George Santayana, The Absence of Religion in Shakespeare, in Santayana, Interpretations, p. $147-165$.

42. "The life of reason a name for all practical thought and all action justified by its fruits in consciousness ", in George Santayana, The Life of Reason, Charleston, BiblioBazaar, 2008, p. 14.

43. "No atheism is so terrible as the absence of an ultimate ideal ", George Santayana, The Sense of Beauty, Being the Outline of Aesthetic Theory, New York, Dover Publications, Inc. p. 160. 
et d'enthousiasme, la raison est un "inviolate principle " qui relie l'ordre parfait aux choses qui existent naturellement. Il considère que la religion est une force variable qui dirige toujours l'homme vers quelque chose d'éternel. C'est la raison pour laquelle la religion n'est pas capable de créer le sentiment de l'harmonie éternelle dans l'esprit humain. "Mais, après tout, pourquoi l'homme a-t-il besoin de la religion ${ }^{44}$ ? ", s'interroge Santayana dans son essai Natural and Ultimate Religion. Parce que la vie est " une ambition crue et ignorante: un amour-propre aveugle empli de toutes sortes d'auto-contradictions, de souffrances et de crimes. C'est exactement de cela qu'une vraie religion viendrait nous délivrer ${ }^{45}$ ". Par conséquent, la religion est une aide naturelle qui nous fait miroiter le réconfort de l'espoir et permet d'obtenir de l'aide en toutes circonstances.

Santayana insiste sur le rôle déterminant de l'intuition dans la religion. Pour lui, Dieu n'est pas une réalité existante mais l'incarnation des aspirations et des envies les plus élevées. En lisant ses écrits, nous pouvons penser qu'aux yeux de Santayana l'essence de la religion est d'offrir un autre monde à l'homme, le monde imaginaire de l'harmonie et de la perfection. C'est pourquoi il pense que la religion et la poésie ont la même racine. Il évoque les anges et les saints comme des symboles significatifs et merveilleux qui jouent un rôle important dans l'imagination de l'homme. Mais ils sont de purs symboles, importants pour le rituel, et ils lient l'homme beaucoup plus fortement à la religion que la simple pensée. Pour cette raison, il avait le sentiment que le protestantisme a rejeté la religion. Joseph Ratner a mentionné dans son étude parue en $1923^{46}$ que selon les observations de Santayana, le rituel a une plus grande importance pour l'homme que le dogme. Pour en donner un exemple significatif, nous pouvons avoir recours de nouveau à Matthew Arnold: Ratner met en parallèle avec les pensées de Santayana l'œuvre d'Arnold intitulée Culture and Anarchy où, en faisant référence à Joubert, celui-ci écrit: "Ma pensée ne sera pas plus claire parce que des milliers d'autres pensent comme moi, mais j'adorerai avec plus d'émotions en communion avec des milliers d'autres ${ }^{47}$. "

Dans Reason in Religion Santayana explique qu'il n'y a pas de moment plus critique dans la vie de l'homme ou d'une nation que lorsque son estime de soi est blessée et qu'il devient prisonnier de sa vanité. Santayana a reconnu l'importance philosophique du moment critique quand le désespoir entraîne des conséquences irrationnelles. Il mentionne deux sortes d'irrationalité, le fanatisme et le mysticisme. Santayana affirme que le fanatique a une vue trop étroite et

44. George Santayana, Natural and Ultimate Religion, in Daniel Cory (éd.), The Birth of Reason and Other Essays by George Santayana, New York, Columbia University Press, 1968, p. 68-69.

45. "Crude, ignorant ambition: a blind self-assertion big with every sort of self-contradiction, agony and crime. It is precisely that from which a veritable religion would come to redeem us ", ibid.

46. Joseph Ratner, " George Santayana’s Theory of Religion », p. 471, The Journal of Religion 3, $\mathrm{n}^{\circ}$ 5, p. 458-475.

47. "It does not help me to think a thing more clearly that thousands of other people are thinking the same; but it does help me to worship with more emotion that thousands of other people are worshipping with me ", ibid. 
qu'il est trop violent pour comprendre le monde, tandis que " le mystique est trop sensualiste et voluptueux pour rationaliser ses sentiments ${ }^{48}$ ". Le fanatique, par son agressivité, veut imposer sa volonté aux autres, il exige une croyance absolue. Il considère sa propre vérité comme la vérité absolue. Santayana déclare en 1914 à la fin de son court essai The Logic of Fanaticism: "Ceux qui croient tenir un bien absolu sont nécessairement sans pitié. Une "vérité" un "Salut" une "Kultur" qu'on espère pouvoir diffuser au moyen de guerres et de persécutions doivent être présumés faux ${ }^{49}$ ». Santayana considère comme dangereux l'attachement aux valeurs absolues qui entrave l'ouverture aux opinions divergentes. Au lieu de croire à la détention de la vérité, il préfère la quête de la vérité. Santayana appelle " unwordly religions " le fanatisme et le mysticisme ${ }^{50}$, que les philosophes doivent trouver le moyen de fuir ${ }^{51}$. La 5th International Conference on Santayana, intitulée The Life of Reason in the Age of Terrorism ${ }^{52}$ s'est tenue à l'été 2016, organisée par le Berlin Practical International Philosophy Forum. Chaque exposé s'est interrogé sur le message véhiculé par la philosophie humaniste de Santayana dans notre monde actuel quand le terrorisme menace. Ses écrits de 1913 qui critiquent le nationalisme extrême et dénoncent l'absurdité de la haine à l'encontre des étrangers sont toujours d'actualité et nous donnent à réfléchir ${ }^{53}$. Le colloque, auquel participaient des philosophes pour la plupart pragmatistes, a souhaité réfuter les stéréotypes qui nient l'utilité des questions et des réponses proposées par les intellectuels ${ }^{54}$.

Santayana a reconnu que l'instabilité sociale (social instability) et la confusion morale (moral confusion) ont pour conséquence que les hommes - par peur du chaos - proclament l'importance de l'ordre. La conformité à l'ordre et l'exigence de le maintenir peuvent mener à des décisions politiques extrêmes. Quand on insiste sur la notion d'ordre, le danger de l'extrémisme peut menacer. "Sans aucun doute, le Chaos a existé, et reviendra " proclame Santayana dans Reason in Common Sense. Dans Dominations and Powers il précise véritablement sa critique des stéréotypes liés à la définition du chaos et de l'ordre. Il écrit dans la partie Chaos and Order: "nous appelons désordre tout ordre dans lequel nous ne pouvons pas reconnaître les essences visibles auxquelles nous sommes habitués.

48. "The mystic is a sensualist too rapt and voluptuous to rationalise his sensations ", in Santayana, The Life of Reason, p. 526.

49. " Those who think they have hold on an absolute good must necessarily be ruthless. [...] A 'truth', a 'salvation', a 'Kultur', which wars and persecutions hope to diffuse is presumably spurious ", in George Santayana, "The Logic of Fanaticism ", The New Republic, 28 novembre 1914, http:// jfredmacdonald.com/worldwarone1914-1918/perspectives-14logic-of-fanaticism.html [dernière consultation le 13 juin 2016].

50. Ibid., p. 523.

51. Ibid., p. 527.

52. [http://berlinphilosophyforum.org/conference-program-july-12-14/], dernière consultation le $1^{\mathrm{er}}$ octobre 2017.

53. Santayana, Winds of Doctrine, New York, Charles Scribner's Sons, 1913, p. 3-5.

54. Ma contribution au colloque paraîtra dans les actes du congrès en 2018, chez Brill, sous le titre " "A happy snow-flake dancing in the flaw" - Reflections on the Santayanan alternatives of surviving the dark ages". 
Nous appelons chaos tout ordre qui crée la confusion dans notre esprit ${ }^{55}$ ". Santayana a précédé les courants philosophiques contemporains qui essaient d'inciter l'homme à se préparer au chaos, aux contingences et à la possibilité du changement continu. Santayana présente une similitude évidente avec la perception des pragmatistes comme par exemple avec Rorty. Selon Santayana, si nous voulons éviter la peur de la confusion morale, nous devons nous fier uniquement à notre intelligence, c'est un milieu parfait pour l'épreuve de la " Life of Reason ", car c'est une méthode en soi par laquelle nous décidons nos propres projets et nos principes. La religion aussi est capable de donner une signification aux choses qui paraissent chaotiques, mais selon Santayana nous devons éviter de réfléchir au moyen des catégories d'ordre absolu ou de vérité absolue parce qu'elles servent uniquement à nous épargner la folie. À vouloir nous persuader que l'ordre est la nature des choses, nous nous étonnons de nous trouver face à des situations inhabituelles et inattendues. Avoir des valeurs intérieures est la seule possibilité pour bannir le chaos apparent autour de nous. Santayana ne pouvait pas croire aux systèmes construits, le doute, l'attitude philosophique la plus importante, était trop fort chez lui. De mentalité naturaliste, il n'était pas capable de s'adonner à la croyance religieuse, et il a observé de manière critique les systèmes institutionnels de la religion. C'est avec une foi particulière qu'il se rapportait au pouvoir de l'imagination et aux capacités de l'individu qu'on peut déployer par le développement de soi en suivant les pas des Anciens de l'Antiquité. Quand Santayana se nomme " moral relativist ", il pense que ni la " spiritual life ", ni la " life of reason " ne peuvent convenir à tout le monde et en toutes circonstances. Selon John Lachs

the life of reason is a way of comporting oneself in the world, and the spiritual life is not. Spirituality consists of pure intuitions, that is, moments of consciousness focused on essences. [...] The life of reason is an extended pattern of existence embodying purposes and principles, whereas the spiritual life is a discontinuous set of self-enclosed acts of vision ${ }^{56}$.

La philosophie de Santayana est par essence individualiste et est destinée aux personnes résolues à se réaliser elles-mêmes, qui n'ont besoin d'aucune aide extérieure et ne s'accrochent à aucun système d'idées construit par autrui.

55. "We give the name of disorder to any order in which we cannot recognise the visible essence to which we are accustomed. Chaos is a name for any order that produces confusion in our minds ", in George Santayana, Dominations and Powers: Reflections on Liberty, Society, and Government, New Brunswick (USA) / London (UK), Transaction Publishers, 1995, p. 33.

56. "The life of reason est une manière de se comporter dans le monde, contrairement à la spiritual life qui consiste en intuitions pures, des moments de conscience qui se concentrent sur les essences. [...] The Life of reason est un large modèle d'existence qui incarne des buts et des principes, tandis que the spiritual life est un ensemble discontinu d'actes de vision clos sur eux-mêmes ", in John Lachs, On Santayana, Thomson, Wadsworth, 2006, p. 60. 


\section{Metanoia laïque}

Vers ses trente ans Santayana s'est trouvé en proie à une crise existentielle. Plus tard, il a appelé metanoia ce tournant de sa vie au cours duquel il a réalisé qu'on le regardait différemment dans son entourage et qu'il ne pouvait plus se considérer comme étant aussi jeune que ses disciples. Il est devenu professeur uniquement dans le but de subvenir à ses besoins mais c'est pour l'apprentissage qu'il s'est senti une vocation. Il serait volontiers resté étudiant s'il avait eu le choix. Il était inspiré par la curiosité des étudiants, par leurs loisirs, par leurs groupes d'amis. Il a ressenti un choc énorme quand il s'est avisé du fait que, sa jeunesse passée, il serait désormais à l'écart de leur milieu ${ }^{57}$. Sa peine s'est approfondie quand son arrière-plan familial est devenu instable, à la mort de son père et après le mariage de sa sœur qui a déménagé en Espagne. Il semble en lisant ses lettres et son autobiographie qu'il n'ait rien vu venir des nouvelles inattendues et douloureuses de sa famille. Il ne put éviter la dépression quand il a appris le décès de son plus cher disciple, son ami le plus proche, Warwick Potter qui a perdu la vie des suites du choléra, lors d'une croisière en France. La vie quotidienne a pris une autre apparence pour lui, il s'est vraiment éloigné de la réalité. "Je me suis rendu compte que ce n'est pas mon monde, seulement celui des autres ${ }^{58}$ " a-t-il écrit. Sa jeunesse insouciante s'est envolée d'un seul coup : " J'ai rompu, à mon corps défendant mais irrémédiablement, avec l'Espagne, l'Angleterre, l'Europe, ma jeunesse et ma religion ${ }^{59}$. "

\section{Conclusion}

Si je voulais conclure sur la vision incroyablement complexe de Santayana sur la religion, je soulignerais qu'au lieu d'accepter une religion institutionnalisée, il s'est focalisé sur la nature de sa propre vie spirituelle et il a essayé de trouver des réponses rationnelles aux autres aspirations et motivations religieuses. Sa théorie de l'essence et son ouvrage tardif The Realm of Spirit justifient sa dévotion à la vie spirituelle. Sa religion laïque était esthétique et rationnelle. Santayana a glorifié l'imagination humaine et, dans ses écrits, il a exprimé son aversion envers la nature de l'homme fanatique et l'étroitesse d'esprit. Il ne pouvait pas s'abandonner aux sentiments religieux traditionnels (ainsi qu'il l'a formulé dans un de ses poèmes, il ne pouvait pas bouger de la porte de l'église) mais il a admis le pouvoir de la religion dans les situations désespérées. Connaissant ses pensées particulières sur la religion, il peut paraître incroyable qu'en 1941, il soit entré à la Clinica della Piccola Compagnia di Maria, un hospice géré par un ordre de religieuses catholiques à Rome. Il y a vécu pendant onze ans jusqu'à sa mort. Santayana a raconté son histoire insolite sur cette admission à Irving Singer qui

57. Santayana, My host the world, p. 7-8.

58. Ibid, p. 109.

59. Ibid, p. 8. 
l'a relatée dans son livre. Santayana a clairement exprimé au couvent qu'il était catholique non pratiquant. Ainsi qu'il l'a raconté à Singer:

They sent me priests to try to convert me, and I argued with them. They went away baffled: the old professor knew more about it than they did! But the Mother Superior and some of the nuns are nice, and I let them leave a Bible in my room. Now I'm worried that at the end I may have a protracted illness, or be in a coma for some time, and they'll be standing around waiting for a last-minute conversion. It can be awfully embarrassing ${ }^{60}$.

Ces notes illustrent de manière éloquente l'ambiguïté qui a caractérisé sa position vis-à-vis de la religion. On pourrait conclure que chez Santayana on peut voir très clairement, et c'est ici que mes réflexions rejoignent celles de l'introduction, que l'approche consistant à voir en la philosophie un mode de vie éveille inévitablement des interrogations religieuses qui peuvent se cristalliser de manières variées (sous la forme de religion au sens étroit ou encore sous forme de poésie). Une vie sans examen, pour reprendre la célèbre formule de Socrate, ne vaut pas la peine d'être vécue; mais une vie avec examen ne peut selon Santayana que s'ouvrir vers la spiritualité. En revanche, et c'est peut-être l'apport précieux de sa pensée à la discussion contemporaine, une vie avec examen fait forcément barrage à des vues du monde "systémiques", absolutisantes, totalitaires qui sont à l'origine de tout fanatisme. Le modèle que nous donne Santayana est ainsi, certes, tout sauf confortable, puisqu'il exige l'examen constant, la mise en question continue, mais il permet de maintenir ouvert un horizon spirituel, tout en excluant toute forme de radicalité et de fanatisme. En somme, la religion reste un point de fuite indispensable, mais qui ne doit pas échapper à l'examen critique ; l'orientation spirituelle nécessite sa propre auto-critique pour ne jamais dériver vers le fanatisme et l'obscurantisme.

Article traduit par Eva Rouault

60. "Elles m’ont envoyé des prêtres pour essayer de me convertir et j'ai discuté avec eux. Ils sont partis déconcertés: le vieux professeur en savait plus qu'eux! Mais la Mère Supérieure et quelques religieuses étaient gentilles et je les ai laissées déposer une bible dans ma chambre. Maintenant j'ai peur qu'à la fin je subisse peut-être une longue maladie ou sois dans le coma pour un certain temps et qu'elles restent là à attendre la conversion de dernière minute. Cela peut être terriblement embarrassant ", in Singer, George Santayana, Literary Philosopher, p. 20.

Finalement Santayana a souhaité être enseveli dans une terre non consacrée, affirmant son naturalisme jusqu'à la fin. Le consulat espagnol à Rome n'a pas permis son enterrement dans le cimetière réservé aux criminels, c'est pourquoi Santayana a été enterré au "Panteon de la Obra pia espagnola ". Nous nous accordons pour reconnaître que c'était une solution acceptable dans le cas de cet homme qui n'a jamais renoncé à sa nationalité espagnole mais que c'était une offense faite au naturaliste, à " l'esthétique catholique ". 